\title{
Study of nonlinear Pachpatte's inequalities on time scales
}

\author{
Sarah Sarfaraz ${ }^{1}$, Naveed Ahmad ${ }^{1 *}$ (D) and Ghaus ur Rahman²
}

${ }^{\text {*Correspondence: }}$

naveedahmad@iba.edu.pk

${ }^{1}$ Department of Mathematics, Institute of Business Administration

(IBA), Karachi, Pakistan

Full list of author information is

available at the end of the article

\begin{abstract}
In this paper, we develop a fundamental dynamic inequality, a generalization of comparison theorem and reproduce the proofs of some nonlinear integral Pachpatte's inequalities by using their continuous analogue. We also unify and extend these improved integral Pachpatte's inequalities and their corresponding discrete analogues on arbitrary time scales. The results are used to make qualitative analysis of higher order dynamic equations.
\end{abstract}

MSC: 35A23; 26E70; 34N05

Keywords: Integral Pachpatte's inequalities; Time scales; Dynamic equations

\section{Introduction}

Modeling of some real world problem requires using a dynamic system which involves both discrete and continuous times. This is natural to see whether it is feasible to give a framework which permits us to incorporate both dynamic systems simultaneously so that one can obtain some insight and a better understanding of the subtle differences of discrete and continuous domains. To answer this, a theory was formulated by Hilger [9]. The main objective of dynamic equations on time scales is that they build bridges between continuous and discrete cases. Later on this theory was developed by many researchers $[3,4]$.

Time scales calculus has many applications in applied as well as in pure mathematics. In pure mathematics time scales calculus has been applied in mathematical inequalities to unify discrete and continuous versions of inequalities. In the last few years a lot of work has been done to unify and extend linear and nonlinear integral inequalities on time scales $[1,8,11,19-22]$. These integral inequalities are used in many applications for the boundedness, uniqueness, etc. of the solutions of different dynamic equations $[6,12,13,15]$.

Pachpatte's inequalities on time scale, both linear and nonlinear, have also been a subject of discussion for quite some time. These Pachpatte's inequalities have been developed by several authors [2, 7, 10, 12], and [14]. Bohner formulated a number of dynamic inequalities that basically rely on Gronwall's inequality [2]. In the literature these kinds of inequalities are known as Pachpatte type inequalities. Also, the authors of the manuscript [8] expounded some nonlinear integral inequalities on time scales, which unified and extended some inequalities established by Pachpatte. To illustrate the theoretical results, it has been shown that the obtained inequalities can be used as important tools in the

(c) The Author(s) 2019. This article is distributed under the terms of the Creative Commons Attribution 4.0 International License (http://creativecommons.org/licenses/by/4.0/), which permits unrestricted use, distribution, and reproduction in any medium, provided you give appropriate credit to the original author(s) and the source, provide a link to the Creative Commons license, and indicate if changes were made. 
study of certain properties of dynamic equations on time scales. Assuming arbitrary time scales, the authors of [14] established some new inequalities of Pachpatte type, incorporating two nonlinear integral terms, which are themselves the generalizations and refinements of many existing results. These obtained results played a vital role in studying some classes of integral and integro-differential equations. Moreover, this approach has various applications in performing the stability analysis of some classes of dynamical systems on time scales. The authors in [18] used some dynamic inequalities to show basic qualitative properties of solutions of a certain nonlinear integrodifferential equation on time scales. The main tools used in the analysis are based on the applications of the Banach fixed point theorem and an inequality with explicit estimate on time scales.

In this paper we present a number of dynamic inequalities that are essentially based on Gronwall's inequality. Most of these inequalities are also known as being of Pachpatte type. In the present assertion, nonlinear Pachpatte's inequalities that we focus on are quite different from the existing ones. Secondly, in the existing theory about Pachpatte's inequalities the authors utilize basic comparison theorem to establish most of the results. But in our case it is not helpful and needs a generalization in the form of fundamental dynamic inequality (see Theorem 3.1) to achieve our goal. Finally, from the application point of view the results are applicable to analyze the qualitative properties of solutions of first order as well as higher order dynamic equations.

The setup of this paper is as follows: Sect. 2 is confined to the basic definitions and preliminary results of calculus on time scales (for further understanding, see [3, 4], and [5]). In Sect. 3 a fundamental dynamic inequality (see Theorem 3.1) is established on an arbitrary time scale. From it we extract the fundamental integral inequality which helps us to reproduce the proofs of nonlinear integral Pachpatte's inequalities ([16], Theorems 2.7.12.7.4). Section 4 is devoted to our main results, in which we accomplished some nonlinear dynamic Pachpatte's inequalities on general time scales. Lastly, we utilize them to study the bounds of the solutions, asymptotic property of the solutions, and the rate at which the solution is growing, of higher order dynamic equations under a few certain suitable conditions.

\section{Calculus on time scales}

A time scale $\mathbb{T}$ is defined as a nonempty arbitrary closed subset of the real numbers $\mathbb{R}$. The following definitions and theorems are referred from [3, 4], and [5]. The proofs of the theorems given below can also be found in the mentioned references.

Definition 2.1 Suppose $\mathbb{T}$ is a time scale. The forward jump operator $\sigma$ is defined as $\sigma(\tau):=\inf \{s \in \mathbb{T}: s>\tau\}$, where $\sigma: \mathbb{T} \rightarrow \mathbb{T}$ for $\tau \in \mathbb{T}$; whereas the backward jump operator $\rho: \mathbb{T} \rightarrow \mathbb{T}$ is defined in a similar manner by $\rho(\tau):=\sup \{s \in \mathbb{T}: s<\tau\}$.

From the preceding definition, it can be said that if $\sigma(\tau)>\tau$, then $\tau$ is right-scattered; however, if $\rho(\tau)<\tau$, then $\tau$ is left-scattered. Also $\sigma(\tau)=\tau$ if $\mathbb{T}$ has a maximum $\tau$ (i.e., $\inf \phi=\sup \mathbb{T})$. Similarly, $\rho(\tau)=\tau$ if $\mathbb{T}$ has a minimum $\tau(\operatorname{or} \sup \phi=\inf \mathbb{T})$. The points which are both right-scattered and left-scattered at once are called isolated. Moreover, if $\tau<\sup \mathbb{T}$ and $\sigma(\tau)=\tau$, then $\tau$ is a right-dense point, and if $\tau>\inf \mathbb{T}$ and $\rho(\tau)=\tau$, then $\tau$ is leftdense. The simultaneously right-dense and left-dense points are known as dense. $\mu(\tau)$ is the graininess function determined by $\mu(\tau):=\phi(\tau)-\tau$, where $\mu(\tau): \mathbb{T} \rightarrow[0, \infty)$. If $\mathbb{T}$ contains a left-scattered maximum element $\tau_{m}$, then $\mathbb{T}^{k}=\mathbb{T}-\left\{\tau_{m}\right\}$; otherwise $\mathbb{T}^{k}=\mathbb{T}$. 
Definition 2.2 The Delta derivative $\mathrm{f}^{\Delta}(\tau)$ of $\mathrm{f}$ at $\tau$, where $\mathrm{f}: \mathbb{T} \rightarrow \mathbb{R}$ and $\tau \in \mathbb{T}^{k}$, is a number such that for every $\epsilon>0$ there exists a neighborhood $U$ with

$$
\left|[\mathrm{f}(\sigma(\tau))-\mathrm{f}(s)]-\mathrm{f}^{\Delta}(\tau)[\sigma(\tau)-s]\right| \leq \epsilon|\sigma(\tau)-s| \quad \text { for all } s \in U
$$

If $\mathbb{T}=\mathbb{Z}$, then $\mathrm{f}^{\Delta}$ is the forward difference $\Delta \mathrm{f}$ (defined by $\Delta \mathrm{f}(\tau)=\mathrm{f}(\tau+1)-\mathrm{f}(\tau)$ ); whereas $\mathrm{f}^{\Delta}$ becomes the usual derivative $\mathrm{f}^{\prime}$ if $\mathbb{T}=\mathbb{R}$.

Theorem 2.3 Suppose $\mathrm{f}, \mathrm{g}: \mathbb{T} \rightarrow \mathbb{R}$ and $\tau \in \mathbb{T}^{k}$. The following results arise:

(i) If $\tau$ is right-scattered and $\mathrm{f}$ is continuous at $\tau$, subsequently $\mathrm{f}$ is differentiable at $\tau$ and $\mathrm{f}^{\Delta}(\tau)$ is given by

$$
\mathrm{f}^{\Delta}(\tau)=\frac{\mathrm{f}(\sigma)(\tau)-\mathrm{f}(\tau)}{\mu(\tau)} .
$$

(ii) If $\tau$ is right-dense, then

$$
\mathrm{f}^{\Delta}(\tau)=\lim _{s \rightarrow \tau} \frac{\mathrm{f}(\tau)-\mathrm{f}(s)}{\tau-s} .
$$

(iii) If $\mathrm{f}$ is differentiable at $\tau$, then $\mathrm{f}$ is continuous at $\tau$.

(iv) If $\mathrm{f}$ is differentiable at $\tau$, then

$$
\mathrm{f}^{\sigma}(\tau)=\mathrm{f}(\tau)+\mu(\tau) \mathrm{f}^{\Delta}(\tau),
$$

where $\mathrm{f}^{\sigma}:=\mathrm{f} \circ \sigma$.

(v) If $\mathrm{f}$ and $\mathrm{g}$ are differential at $\tau$, then so is $\mathrm{fg}$ with

$$
(\mathrm{fg})^{\Delta}(\tau)=\mathrm{f}^{\Delta}(\tau) \mathrm{g}(\tau)+\mathrm{f}^{\sigma}(\tau) \mathrm{g}^{\Delta}(\tau) .
$$

Definition 2.4 A right-dense continuous or rd-continuous function $f: \mathbb{T} \rightarrow \mathbb{R}$ is a function which is continuous at the right-dense points in $\mathbb{T}$ and its left-sided limits exist (finite) at left-dense points in $\mathbb{T}$. The set of all $\mathrm{rd}$-continuous functions is denoted by $C_{\mathrm{rd}}$, while the set of functions that are differentiable and whose derivative is rd-continuous is denoted by $C_{\text {rd }}^{1}$.

Theorem 2.5 Every $r d$-continuous function has an antiderivative. Let $\tau_{0} \in \mathbb{T}$, then $\mathrm{f}$ defined by

$$
\mathrm{f}(\tau):=\int_{\tau_{0}}^{\tau} \mathrm{f}(x) \Delta x \quad \text { for } \tau \in \mathbb{T}
$$

is an antiderivative of $\mathrm{f}$.

Theorem 2.6 If $\mathrm{f} \in C_{\mathrm{rd}}$ and $\tau \in \mathbb{T}^{k}$, then

$$
\int_{\tau_{0}}^{\sigma(\tau)} \mathrm{f}(x) \Delta x=\mu(\tau) \mathrm{f}(\tau)
$$


Theorem 2.7 If $a$ and $b \in \mathbb{T}$ and $\mathrm{f} \in C_{\mathrm{rd}}$ provided $\mathrm{f}(\tau) \geq 0$ for all $a \leq \tau \leq b$, then

$$
\int_{a}^{b} \mathrm{f}(\tau) \Delta \tau \geq 0
$$

Theorem 2.8 Let $\mathrm{f}: \mathbb{R} \rightarrow \mathbb{R}$ be continuously differentiable and $\mathrm{g}: \mathbb{R} \rightarrow \mathbb{R}$ be continuous, $\mathrm{g}: \mathbb{T} \rightarrow \mathbb{R}$ is delta differentiable on $\mathbb{T}$. Then there exists $c \in[\tau, \sigma(\tau)]$ with

$$
(\mathrm{f} \circ \mathrm{g})^{\Delta}(\tau)=\mathrm{f}^{\prime}(\mathrm{g}(c)) \mathrm{g}^{\Delta}(\tau)
$$

Definition 2.9 A function $p: \mathbb{T} \rightarrow \mathbb{R}$ is regressive if $1+\mu(\tau) p(\tau) \neq 0$ for all $\tau \in \mathbb{T}$. The set of all regressive and rd-continuous functions is denoted by $R$.

The set of all positive regressive functions is $R^{+}=\{p \in R: 1+\mu(\tau) p(\tau)>0$ for all $\tau \in \mathbb{T}\}$.

If $p, q \in R$, then we define

$$
\begin{aligned}
& p(\tau) \oplus q(\tau)=p(\tau)+q(\tau)+\mu(\tau) p(\tau) q(\tau), \\
& \ominus q(\tau)=-\frac{q(\tau)}{1+\mu q(\tau)}, \quad \text { and } \\
& p(\tau) \ominus q(\tau)=p(\tau) \oplus(\ominus q(\tau)) .
\end{aligned}
$$

Theorem 2.10 If $p \in C_{\mathrm{rd}}$ and $p \in R$, where $p: \mathbb{T} \rightarrow \mathbb{R}$, then the exponential function $e_{p}\left(\cdot, \tau_{0}\right)$, for all $\tau_{0} \in \mathbb{T}$, is the unique solution of the initial value problem

$$
y^{\Delta}=p(\tau) y, \quad y\left(\tau_{0}\right)=1
$$

The following theorem exhibits some properties related to an exponential function.

Theorem 2.11 If $p, q \in R$, then

(i) $e_{p}(\tau, \tau)=1$ and $e_{0}(\tau, s)=1$;

(ii) $e_{p}(\sigma(\tau), s)=(1+\mu(\tau) p(\tau)) e_{p}(\tau, s)$;

(iii) $\frac{1}{e_{p}(\tau, s)}=e_{\ominus p}(\tau, s)=e_{p}(s, \tau)$;

(iv) $\frac{e_{p}(\tau, s)}{e_{q}(\tau, s)}=e_{p \ominus q}(\tau, s)$;

(v) $e_{p}(\tau, s) e_{q}(\tau, s)=e_{p \oplus q}(\tau, s)$;

(vi) If $p \in R^{+}$, then $e_{p}\left(\tau, \tau_{0}\right)>0$ for all $\tau \in \mathbb{T}$.

Example 2.12 We note that, if $\mathbb{T}=\mathbb{R}, p: \mathbb{R} \rightarrow \mathbb{R}$ is a continuous function and $s, \tau \in \mathbb{R}$, the exponential function is given by

$$
e_{p}(\tau, s)=e^{\int_{s}^{\tau} p(x) d x}
$$

If $p$ is a constant $\alpha \in \mathbb{R}$, the exponential function becomes

$$
e^{\int_{s}^{\tau} \alpha d x}=e^{\alpha(\tau-s)}
$$

if $s=0$, then we get

$$
e^{\alpha(\tau-s)}=e^{\alpha \tau}
$$


We also give the exponential function for $\mathbb{T}=\mathbb{Z}$ when $p: \mathbb{Z} \rightarrow \mathbb{R}$ is a sequence satisfying $p(\tau) \neq-1$ for all $\tau \in \mathbb{Z}$ and $s, \tau \in \mathbb{Z}$ with $s<\tau$ as

$$
e_{p}(\tau, s)=\prod_{x=s}^{\tau-1}[1+p(x)]
$$

When $p=\alpha$, where $\alpha \neq-1$ is a constant, then

$$
\prod_{x=s}^{\tau-1}[1+p(x)]=(1+\alpha)^{\tau-s}
$$

if $s=0$,

$$
(1+\alpha)^{\tau-s}=(1+\alpha)^{\tau} .
$$

Given notation will be followed throughout the text for ease of reading:

$$
\tau_{0} \in \mathbb{T}, \quad \mathbb{T}_{0}=\left[\tau_{0}, \infty\right) \cap \mathbb{T} .
$$

\section{Fundamental dynamic inequality}

The following dynamic inequality plays a key role in inaugurating the main results of this paper. The inequality is stated as follows.

Theorem 3.1 Let $v(\tau)$ be a positive function defined for $\tau \in \mathbb{T}_{0}$, let $b(\tau)$ and $k(\tau)$ be nonnegative functions defined for $\tau \in \mathbb{T}_{0}$, and let $b(\tau)$ be regressive and $\alpha \geq 0, \alpha \neq 1$ be a constant. If

$$
v^{\Delta}(\tau) \leq b(\tau) v(\tau)+k(\tau) v^{\alpha}(\tau)
$$

for $\tau \in \mathbb{T}_{0}$ and $v^{1-\alpha}\left(\tau_{0}\right)+(1-\alpha) \int_{s=\tau_{0}}^{\tau} k(s)\left(e_{\ominus b}\left(\sigma(s), \tau_{0}\right)\right)^{1-\alpha} \Delta s>0$ for $\tau \in \mathbb{T}_{0}$, then

$$
v(\tau) \leq e_{b}\left(\tau, \tau_{0}\right)\left\{v^{1-\alpha}\left(\tau_{0}\right)+(1-\alpha) \int_{s=\tau_{0}}^{\tau} k(s)\left(e_{\ominus b}\left(\sigma(s), \tau_{0}\right)\right)^{1-\alpha} \Delta s\right\}^{\frac{1}{1-\alpha}}
$$

for $\tau \in \mathbb{T}_{0}$.

Proof We note that $e_{\ominus b}\left(\tau, \tau_{0}\right)$ satisfies the following equation as in [3]:

$$
e_{\ominus b}^{\Delta}\left(\tau, \tau_{0}\right)=-b(t) e_{\ominus b}\left(\sigma(\tau), \tau_{0}\right), \quad e_{\ominus b}\left(\tau_{0}, \tau_{0}\right)=1 .
$$

Now multiplying (1) by $e_{\ominus b}\left(\sigma(\tau), \tau_{0}\right)$, we obtain

$$
v^{\Delta}(\tau) e_{\ominus b}\left(\sigma(\tau), \tau_{0}\right)-e_{\ominus b}\left(\sigma(\tau), \tau_{0}\right) b(\tau) v(\tau) \leq e_{\ominus b}\left(\sigma(\tau), \tau_{0}\right) k(\tau) v^{\alpha}(\tau)
$$

Using (3) in (4), we get

$$
\left(v(\tau) e_{\ominus b}\left(\tau, \tau_{0}\right)\right)^{\Delta} \leq k(\tau) v^{\alpha}(\tau) e_{\ominus b}\left(\sigma(\tau), \tau_{0}\right) .
$$


Set $q=1-\alpha$ and use the following mean value theorem:

$$
(\mathrm{f} \circ \chi)^{\Delta}=\mathrm{f}^{\prime}(x) \chi^{\Delta}(\tau)
$$

where $x$ lies between $\chi(\tau)$ and $\chi(\sigma(\tau))$.

Take $\chi(\tau)=v(\tau) e_{\ominus b}\left(\tau, \tau_{0}\right)>0$, then $\chi(\sigma(\tau))=v(\sigma(\tau)) e_{\ominus b}\left(\sigma(\tau), \tau_{0}\right)$.

From (6) we get

$$
\left[\left(v(\tau) e_{\ominus b}\left(\tau, \tau_{0}\right)\right)^{q}\right]^{\Delta}=q x^{-\alpha}\left[v(\tau) e_{\ominus b}\left(\tau, \tau_{0}\right)\right]^{\Delta} .
$$

For $\mathrm{f}(\tau)=\tau^{q} \mathrm{f}: \mathbb{R} \rightarrow \mathbb{R}$ and $\chi(\tau): \mathbb{T} \rightarrow \mathbb{R}$. For the estimation of the value of $x$ in equation (7), we have considered the following two factual cases:

Case I: If $x \in(\chi(\tau), \chi(\sigma(\tau)))$, then $x>v(\tau) e_{\ominus b}\left(\tau, \tau_{0}\right) \geq v(\tau) e_{\ominus b}\left(\sigma(\tau), \tau_{0}\right)$.

Case II: If $x \in(\chi(\sigma(\tau)), \chi(\tau))$, then $x>v(\sigma(\tau)) e_{\ominus b}\left(\sigma(\tau), \tau_{0}\right) \geq v(\tau) e_{\ominus b}\left(\sigma(\tau), \tau_{0}\right)$.

Depending on the value of $q$, we have two scenarios for the above mentioned two cases:

(i) $q>0(0 \leq \alpha<1)$.

Since

$$
x \geq v(\tau) e_{\ominus b}\left(\sigma(\tau), \tau_{0}\right),
$$

then

$$
q x^{-\alpha} k(\tau) v^{\alpha}(\tau) e_{\ominus b}\left(\sigma(\tau), \tau_{0}\right) \leq q\left[v(\tau) e_{\ominus b}\left(\sigma(\tau), \tau_{0}\right)\right]^{-\alpha} k(\tau) v^{\alpha}(\tau) e_{\ominus b}\left(\sigma(\tau), \tau_{0}\right) .
$$

Also, (5) can be written as

$$
q x^{-\alpha}\left(v(\tau) e_{\ominus b}\left(\tau, \tau_{0}\right)\right)^{\Delta} \leq q x^{-\alpha} k(\tau) v^{\alpha}(\tau) e_{\ominus b}\left(\sigma(\tau), \tau_{0}\right),
$$

so (7) becomes

$$
\left[\left(v(\tau) e_{\ominus b}\left(\tau, \tau_{0}\right)\right)^{q}\right]^{\Delta} \leq q x^{-\alpha} k(\tau) v^{\alpha}(\tau) e_{\ominus b}\left(\sigma(\tau), \tau_{0}\right) .
$$

Thus, from (8) and (9), it can be acquired that

$$
\left[\left(v(\tau) e_{\ominus b}\left(\tau, \tau_{0}\right)\right)^{q}\right]^{\Delta} \leq q k(\tau)\left[e_{\ominus b}\left(\sigma(\tau), \tau_{0}\right)\right]^{q} .
$$

Setting $\tau=s$ and integrating from $\tau_{0}$ to $\tau$, we get

$$
\left(v(\tau) e_{\ominus b}\left(\tau, \tau_{0}\right)\right)^{q} \leq v^{q}\left(\tau_{0}\right)+q \int_{\tau_{0}}^{\tau} k(s)\left[e_{\ominus b}\left(\sigma(s), \tau_{0}\right)\right]^{q} \Delta s .
$$

Taking $q^{\text {th }}$ root, it follows that

$$
v(\tau) e_{\ominus b}\left(\tau, \tau_{0}\right) \leq\left\{v^{q}\left(\tau_{0}\right)+q \int_{\tau_{0}}^{\tau} k(s)\left[e_{\ominus b}\left(\sigma(s), \tau_{0}\right)\right]^{q} \Delta s\right\}^{\frac{1}{q}} .
$$


It implies that

$$
v(\tau) \leq e_{b}\left(\tau, \tau_{0}\right)\left\{v^{1-\alpha}\left(\tau_{0}\right)+(1-\alpha) \int_{\tau_{0}}^{\tau} k(s)\left[e_{\ominus b}\left(\sigma(s), \tau_{0}\right)\right]^{1-\alpha} \Delta s\right\}^{\frac{1}{1-\alpha}} .
$$

(ii) $q<0(\alpha>1)$.

Again taking

$$
x \geq v(\tau) e_{\ominus b}\left(\sigma(\tau), \tau_{0}\right),
$$

we obtain

$$
q x^{-\alpha} k(\tau) v^{\alpha}(\tau) e_{\ominus b}\left(\sigma(\tau), \tau_{0}\right) \geq q\left[v(\tau) e_{\ominus b}\left(\sigma(\tau), \tau_{0}\right)\right]^{-\alpha} k(\tau) v^{p}(\tau) e_{\ominus b}\left(\sigma(\tau), \tau_{0}\right) .
$$

Now (5) will take the following form:

$$
q x^{-\alpha}\left(v(\tau) e_{\ominus b}\left(\tau, \tau_{0}\right)\right)^{\Delta} \geq q x^{-\alpha} k(\tau) v^{\alpha}(\tau) e_{\ominus b}\left(\sigma(\tau), \tau_{0}\right)
$$

and (7) changes into

$$
\left[\left(v(\tau) e_{\ominus b}\left(\tau, \tau_{0}\right)\right)^{q}\right]^{\Delta} \geq q x^{-\alpha} k(\tau) v^{\alpha}(\tau) e_{\ominus b}\left(\sigma(\tau), \tau_{0}\right) .
$$

Hence, from (11) and (12), we obtained

$$
\left[\left(v(\tau) e_{\ominus b}\left(\tau, \tau_{0}\right)\right)^{q}\right]^{\Delta} \geq q k(\tau)\left[e_{\ominus b}\left(\sigma(\tau), \tau_{0}\right)\right]^{q} .
$$

Putting $\tau=s$ and taking integral from $\tau_{0}$ to $\tau$, we attain

$$
\left(v(\tau) e_{\ominus b}\left(\tau, \tau_{0}\right)\right)^{q} \geq v^{q}\left(\tau_{0}\right)+q \int_{\tau_{0}}^{\tau} k(s)\left[e_{\ominus b}\left(\sigma(s), \tau_{0}\right)\right]^{q} \Delta s .
$$

On applying $q^{\text {th }}$ root on both sides, it results into

$$
v(\tau) e_{\ominus b}\left(\tau, \tau_{0}\right) \leq\left\{v^{q}\left(\tau_{0}\right)+q \int_{\tau_{0}}^{\tau} k(s)\left[e_{\ominus b}\left(\sigma(s), \tau_{0}\right)\right]^{q} \Delta s\right\}^{\frac{1}{q}} .
$$

It infers that

$$
v(\tau) \leq e_{b}\left(\tau, \tau_{0}\right)\left\{v^{1-\alpha}\left(\tau_{0}\right)+(1-\alpha) \int_{\tau_{0}}^{\tau} k(s)\left[e_{\ominus b}\left(\sigma(s), \tau_{0}\right)\right]^{1-\alpha} \Delta s\right\}^{\frac{1}{1-\alpha}}
$$

Thus the proof is completed.

On an arbitrary time scale $\mathbb{T}$, one can see that the inequality proved above is a generalization of some existing results.

\section{Remark 3.2}

- If $\mathbb{T}=\mathbb{Z}$, Theorem 3.1 implies Theorem 2.3.4 established by Pachpatte in [17]. 
- For an arbitrary time scale, if $\alpha=0$, Theorem 3.1 reduces to Theorem 5.4, also known as Comparison Theorem, proved by Agarwal, Bohner, and Peterson [1].

Corollary 3.3 Let $\mathbb{T}=\mathbb{R}$ and assume $v(\tau)$ is a positive function defined for $\tau \in \mathbb{R}$, let $b(\tau)$ and $k(\tau)$ be nonnegative functions defined for $\tau \in \mathbb{R}$ and $\alpha \geq 0, \alpha \neq 1$ be a constant. If

$$
v^{\prime}(\tau) \leq b(\tau) v(\tau)+k(\tau) v^{\alpha}(\tau)
$$

for $\tau \in \mathbb{R}$ and $v^{1-\alpha}\left(\tau_{0}\right)+(1-\alpha) \int_{s=0}^{\tau} k(s) e^{-(1-\alpha) \int_{0}^{s} b(\xi) d \xi} d s>0$ for $\tau \in \mathbb{R}$, then

$$
v(\tau) \leq e^{\int_{0}^{\tau} b(\xi) d \xi}\left\{v^{1-\alpha}\left(\tau_{0}\right)+(1-\alpha) \int_{s=0}^{\tau} k(s) e^{-(1-\alpha) \int_{0}^{\tau} b(\xi) d \xi} d s\right\}^{\frac{1}{1-\alpha}}
$$

for $\tau \in \mathbb{R}$.

Remark 3.4 The next part of this section is related to reproduction of the proofs of nonlinear Pachpatte's inequalities (Theorems 2.7.1-2.7.4) proved by Pachpatte in [16]. These inequalities are reproduced here via Corollary 3.3. Only the proof of Theorem 2.7.1 is given below, the rest of the inequalities can be proved in a similar way.

Theorem 3.5 Let $v$, $\mathrm{f}$, and $\mathrm{g}$ be nonnegative continuous functions on $\mathbb{R}_{+}$and $c>0, \alpha>0$, and $\alpha \neq 1$ be constants, and suppose

$$
v(\tau) \leq \mathrm{c}+\int_{0}^{\tau} \mathrm{f}(s) v(s) d s+\int_{0}^{\tau} \mathrm{f}(s)\left[\int_{0}^{s} \mathrm{~g}(x) v^{\alpha}(x) d x\right] d s
$$

for $\tau \in \mathbb{R}_{+}$.

(i) If $0<\alpha<1$ and $E_{0}(\tau)$ is defined by

$$
E_{0}(\tau)=1+(1-\alpha) \mathrm{c}^{\alpha-1} \int_{0}^{\tau} \mathrm{g}(t)\left(e^{-\int_{0}^{t} \mathrm{f}(s) d s}\right)^{(1-\alpha)} d t,
$$

then

$$
v(\tau) \leq \mathrm{c}\left[1+\int_{0}^{\tau} \mathrm{f}(s)\left(e^{\int_{0}^{s} \mathrm{f}(x) d x}\right)\left(E_{0}(s)\right)^{\left(\frac{1}{1-\alpha}\right)} d s\right]
$$

for $\tau \in \mathbb{R}_{+}$.

(ii) If $1<\alpha<\infty$, then

$$
v(\tau) \leq \mathrm{c}\left[1+\int_{0}^{\tau} \mathrm{f}(s)\left(e^{-\int_{0}^{s} \mathrm{f}(x) d x}\right)\left(\bar{E}_{0}(s)\right)^{\left(\frac{-1}{\alpha-1}\right)} d s\right]
$$

for $\tau \in[0, j]$, where

$$
\bar{E}_{0}(\tau)=1+(1-\alpha) \mathrm{c}^{\alpha-1} \int_{0}^{\tau} \mathrm{g}(t)\left(e^{-\int_{0}^{t} \mathrm{f}(s) d s}\right)^{(1-\alpha)} d t
$$

for $1<\alpha<\infty$ and $j=\sup \left\{\tau \in \mathbb{R}_{+}: \bar{E}_{0}(\tau)>0\right\}$. Furthermore, assume $\bar{E}_{0}(\tau)>0$ for all $\tau \in \mathbb{R}_{+}$, subsequently inequality (18) holds for all $\tau \in \mathbb{R}_{+}$. 
Proof (i) Let $0<\alpha<1$ and let

$$
\chi(\tau)=\mathrm{c}+\int_{0}^{\tau} \mathrm{f}(s) v(s) d s+\int_{0}^{\tau} \mathrm{f}(s)\left[\int_{0}^{s} \mathrm{~g}(x) v^{\alpha}(x) d x\right] d s,
$$

then $\chi(0)=\mathrm{c}, v(\tau) \leq \chi(\tau)$ and

$$
\begin{aligned}
& \chi^{\prime}(\tau) \leq \mathrm{f}(\tau)\left(v(\tau)+\int_{0}^{\tau} \mathrm{g}(x) v^{\alpha}(x) d x\right), \\
& \chi^{\prime}(\tau) \leq \mathrm{f}(\tau)\left(\chi(\tau)+\int_{0}^{\tau} \mathrm{g}(x) \chi^{\alpha}(x) d x\right) .
\end{aligned}
$$

Define a function $\psi(\tau)$ by

$$
\psi(\tau)=\chi(\tau)+\int_{0}^{\tau} \mathrm{g}(x) \chi^{\alpha}(x) d x
$$

then $\psi(0)=\chi(0)=c, \chi(\tau) \leq \psi(\tau), \chi^{\prime}(\tau) \leq \mathrm{f}(\tau) \psi(\tau)$, and

$$
\begin{aligned}
\psi^{\prime}(\tau) & \leq \chi^{\prime}(\tau)+\mathrm{g}(\tau) \chi^{\alpha}(\tau) \\
& \leq \mathrm{f}(\tau) \psi(\tau)+\mathrm{g}(\tau) \psi^{\alpha}(\tau) .
\end{aligned}
$$

Applying Theorem 3.3 to (21) yields

$$
\begin{aligned}
\psi(\tau) & \leq e^{-\int_{0}^{\tau} \mathrm{f}(x) d x}\left\{\mathrm{c}^{1-\alpha}+(1-\alpha) \int_{0}^{\tau} \mathrm{g}(s) \cdot e^{-(1-\alpha) \int_{0}^{s} \mathrm{f}(x) d x} d s\right\}^{\frac{1}{1-\alpha}} \\
& =\mathrm{c} e^{-\int_{0}^{\tau} \mathrm{f}(x) d x}\left\{1+(1-\alpha) \mathrm{c}^{\alpha-1} \int_{0}^{\tau} \mathrm{g}(s) \cdot e^{-(1-\alpha) \int_{0}^{s} \mathrm{f}(x) d x} d s\right\}^{\frac{1}{1-\alpha}} \\
& =\mathrm{c} e^{-\int_{0}^{\tau} \mathrm{f}(x) d x}\left\{E_{0}(\tau)\right\}^{\frac{1}{1-\alpha}} .
\end{aligned}
$$

Using (22) in (19), we get

$$
\chi^{\prime}(\tau) \leq \operatorname{cf}(\tau) e^{-\int_{0}^{\tau} f(x) d x}\left(E_{0}(\tau)\right)^{\frac{1}{1-\alpha}} .
$$

The preceding inequality suggests the approximation for $\chi(\tau)$ such that

$$
\chi(\tau) \leq \mathrm{c}\left[1+\int_{0}^{\tau} \mathrm{f}(s) e^{-\int_{0}^{s} \mathrm{f}(x) d x}\left(E_{0}(s)\right)^{\left(\frac{1}{1-\alpha}\right)} d s\right] .
$$

Now, using (24) and the fact $v(\tau) \leq \chi(\tau)$, we get the required inequality.

(ii) Let $1<\alpha<\infty$. Further moving with similar steps like in the proof of case (i), it is seen that inequalities (22) and (24) hold for $\tau \in[0, j)$, and hence we obtain inequality (18) which holds for $\tau \in[0, j)$. The last conclusion is obvious. Thus the proof is complete.

\section{Nonlinear Pachpatte's inequalities on time scales}

The improved proofs of nonlinear Pachpatte's inequalities (Theorems 2.7.1-2.7.4 in [16]), discussed in Sect. 3, enable us to unify them along with their discrete analogues (Theorems 2.4.1, 2.4.2, 2.4.4, and 2.4.5 in [17]) on an arbitrary time scale. 
Theorem 4.1 Let $u, \mathrm{f}, \mathrm{g} \in C_{\mathrm{rd}}$ and be nonnegative on $\mathbb{T}_{0}$ and $c>0, \alpha>0$, and $\alpha \neq 1$ be constants, and suppose

$$
v(\tau) \leq c+\int_{\tau_{0}}^{\tau} \mathrm{f}(s) u(s) \Delta s+\int_{\tau_{0}}^{\tau} \mathrm{f}(s)\left[\int_{\tau_{0}}^{s} \mathrm{~g}(x) v^{\alpha}(x) \Delta x\right] \Delta s
$$

for $t \in \mathbb{T}_{0}$.

(i) If $0<\alpha<1$ and $E_{0}(\tau)$ is defined by

$$
E_{0}(\tau)=1+(1-\alpha) c^{\alpha-1} \int_{\tau_{0}}^{\tau} \mathrm{g}(t)\left(e_{\ominus f}\left(\sigma(t), \tau_{0}\right)\right)^{(1-\alpha)} \Delta t
$$

then

$$
v(\tau) \leq c\left[1+\int_{\tau_{0}}^{\tau} \mathrm{f}(s) e_{\mathrm{f}}\left(s, \tau_{0}\right)\left(E_{0}(s)\right)^{\left(\frac{1}{1-\alpha}\right)} \Delta s\right]
$$

for $\tau \in \mathbb{T}_{0}$.

(ii) If $1<\alpha<\infty$, then

$$
v(\tau) \leq c\left[1+\int_{\tau_{0}}^{\tau} \mathrm{f}(s) e_{\mathrm{f}}\left(s, \tau_{0}\right)\left(\overline{E_{0}}(s)\right)^{\left(-\frac{1}{\alpha-1}\right)} \Delta s\right]
$$

for $\tau \in\left[\tau_{0}, j\right]$, where

$$
\bar{E}_{0}(\tau)=1+(1-\alpha) c^{\alpha-1} \int_{\tau_{0}}^{\tau} \mathrm{g}(t)\left(e_{\ominus \mathrm{f}}\left(\sigma(t), \tau_{0}\right)\right)^{(1-\alpha)} \Delta t
$$

for $1<\alpha<\infty$ and $j=\sup \left\{\tau \in \mathbb{T}_{0}: \bar{E}_{0}(\tau)>0\right\}$. Furthermore, assume $\bar{E}_{0}(\tau)>0$ for all $t \in \mathbb{T}_{0}$, subsequently inequality (27) still holds for all $\tau \in T_{0}$.

Theorem 4.2 Let $v, \mathrm{f}, \mathrm{g}, h \in C_{\mathrm{rd}}$ and be nonnegative on $\mathbb{T}_{0}$ and $c>0$ be a constant, and suppose

$$
v(\tau) \leq c+\int_{\tau_{0}}^{\tau} \mathrm{f}(s) v(s) \Delta s+\int_{\tau_{0}}^{\tau} \mathrm{g}(s) v(s)\left[v(s)+\int_{\tau_{0}}^{s} h(x) v(x) \Delta x\right] \Delta s
$$

for $\tau \in \mathbb{T}_{0}$. Then

$$
v(\tau) \leq c e_{\left(\mathrm{f}(s)+c g(s)(E(s))^{-1} e_{(\mathrm{f}+h)}\left(s, \tau_{0}\right)\right)}\left(\tau, \tau_{0}\right)
$$

for $\tau \in\left[\tau_{0}, j_{1}\right)$, where

$$
E(\tau)=1-c \int_{\tau_{0}}^{\tau} \mathrm{g}(t) e_{(\mathrm{f}+h)}\left(\sigma(t), \tau_{0}\right) \Delta t
$$

and $j_{1}=\sup \left\{\tau \in \mathbb{T}_{0}: E(\tau)>0\right\}$. Furthermore, assume $E(\tau)>0$ for all $\tau \in \mathbb{T}_{0}$, subsequently inequality (29) still holds for all $\tau \in \mathbb{T}_{0}$. 
Theorem 4.3 Let $v, \mathrm{f}, \mathrm{g}, h$, and $c$ be as in Theorem 4.2 and $\alpha>0, \alpha \neq 1$ be a constant, and suppose

$$
v(\tau) \leq c+\int_{\tau_{0}}^{\tau} \mathrm{f}(s) v(s) \Delta s+\int_{\tau_{0}}^{\tau} \mathrm{g}(s) v^{\alpha}(s)\left[v(s)+\int_{\tau_{0}}^{s} h(x) v(x) \Delta x\right] \Delta s
$$

for $\tau \in \mathbb{T}_{0}$.

(i) If $0<\alpha<1$ and $E_{1}(\tau)$

$$
E_{1}(\tau)=1-\alpha c^{\alpha} \int_{\tau_{0}}^{\tau} \mathrm{g}(t)\left(e_{\ominus(\mathrm{f}+h)}\left(\sigma(t), \tau_{0}\right)\right)^{-\alpha} \Delta t
$$

then

$$
\begin{aligned}
v(\tau) \leq & c e_{\mathrm{f}}\left(\tau, \tau_{0}\right)\left[1+(1-\alpha) c^{\alpha} \int_{\tau_{0}}^{\tau} e_{(\mathrm{f}+h)}\left(s, \tau_{0}\right)\left(E_{1}(s)\right)^{\left(\frac{-1}{\alpha}\right)}\right. \\
& \left.\times \mathrm{g}(s)\left(e_{\ominus \mathrm{f}}\left(\sigma(s), \tau_{0}\right)\right)^{1-\alpha} \Delta s\right]^{\left(\frac{1}{1-\alpha}\right)}
\end{aligned}
$$

for $\tau \in\left[\tau_{0}, j_{2}\right)$, where $j_{2}=\sup \left\{\tau \in \mathbb{T}_{0}: E_{1}(\tau)>0\right\}$.

(ii) If $1<\alpha<\infty$, then

$$
\begin{aligned}
v(\tau) \leq & c e_{\mathrm{f}}\left(\tau, \tau_{0}\right)\left[1-(\alpha-1) c^{\alpha} \int_{\tau_{0}}^{\tau} e_{(\mathrm{f}+h)}\left(s, \tau_{0}\right)\left(\overline{E_{1}(s)}\right)^{\left(\frac{-1}{\alpha}\right)}\right. \\
& \left.\times \mathrm{g}(s)\left(e_{\ominus \mathrm{f}}\left(\sigma(s), \tau_{0}\right)\right)^{1-\alpha} \Delta s\right]^{\frac{-1}{\alpha-1}}
\end{aligned}
$$

where $\tau \in\left[\tau_{0}, j_{3}\right]$, while

$$
\bar{E}_{1}(\tau)=1-\alpha c^{\alpha} \int_{\tau_{0}}^{\tau} \mathrm{g}(t)\left(e_{\ominus(\mathrm{f}+h)}\left(\sigma(t), \tau_{0}\right)\right)^{-\alpha} \Delta t
$$

for $1<\alpha<\infty$ and $j_{3}$ is the supremum of $\tau \in \mathbb{T}_{0}$ for which $\bar{E}_{1}(\tau)$ and the term multiplied by $\operatorname{ce}_{\mathrm{f}}\left(\tau, \tau_{0}\right)$ in (33) are positive. Furthermore, assume (i) $E_{1}(\tau)>0$ and (ii) $\bar{E}_{1}(\tau)>0$ and the term multiplied by ce $e_{\mathrm{f}}\left(\tau, \tau_{0}\right)$ in (33) is positive for all $\tau \in \mathbb{T}_{0}$; subsequently, inequalities (32) and (33) hold respectively for all $\tau \in \mathbb{T}_{0}$.

Remark 4.4 Since the proofs are similar to one another, the proofs of Theorems 4.1-4.3 can be accomplished by following the proof of Theorem 4.5. The details are given below.

Theorem 4.5 Let $v, \mathrm{f}, \mathrm{g}, h$, and $c$ be as in Theorem 4.3, and suppose

$$
v(\tau) \leq c+\int_{\tau_{0}}^{\tau} \mathrm{f}(s) v(s) \Delta s+\int_{\tau_{0}}^{\tau} \mathrm{g}(s) v^{\alpha}(s)\left[v(s)+\int_{\tau_{0}}^{s} h(x) v^{\alpha+1}(x) \Delta x\right] \Delta s
$$

for $\tau \in \mathbb{T}_{0}$.

(i) If $0<\alpha<1$ and $E_{2}(\tau)$ is defined by

$$
E_{2}(\tau)=1-\alpha c^{\alpha} \int_{\tau_{0}}^{\tau}[\mathrm{g}(t)+h(t)]\left(e_{\ominus \mathrm{f}}\left(\sigma(t), \tau_{0}\right)\right)^{-\alpha} \Delta t,
$$


then

$$
\begin{aligned}
v(\tau) \leq & c e_{\mathrm{f}}\left(\tau, \tau_{0}\right)\left[1+(1-\alpha) c^{\alpha} \int_{\tau_{0}}^{\tau} \mathrm{g}(s) e_{\mathrm{f}}\left(s, \tau_{0}\right)\left(E_{2}(s)\right)^{-\frac{1}{\alpha}}\right. \\
& \left.\times\left(e_{\ominus \mathrm{f}}\left(\sigma(s), \tau_{0}\right)\right)^{1-\alpha} \Delta s\right]^{\frac{1}{1-\alpha}}
\end{aligned}
$$

for $\tau \in\left[\tau_{0}, j_{4}\right)$, where $j_{4}=\sup \left\{\tau \in \mathbb{T}_{0}: E_{2}(\tau)>0\right\}$.

(ii) If $1<\alpha<\infty$, then

$$
\begin{aligned}
v(\tau) \leq & c e_{\mathrm{f}}\left(\tau, \tau_{0}\right)\left[1-(\alpha-1) c^{\alpha} \int_{\tau_{0}}^{\tau} \mathrm{g}(s) e_{\mathrm{f}}\left(s, \tau_{0}\right)\left(\overline{E_{2}}(s)\right)^{-\frac{1}{\alpha}}\right. \\
& \left.\times\left(e_{\ominus \mathrm{f}}\left(\sigma(s), \tau_{0}\right)\right)^{1-\alpha} \Delta s\right]^{\frac{-1}{\alpha-1}}
\end{aligned}
$$

where $\tau \in\left[\tau_{0}, j_{5}\right]$ and

$$
\bar{E}_{2}(\tau)=1-\alpha c^{\alpha} \int_{\tau_{0}}^{\tau}[\mathrm{g}(t)+h(t)]\left(e_{\ominus f}\left(\sigma(t), \tau_{0}\right)\right)^{-\alpha} \Delta t
$$

for $1<\alpha<\infty$ and $j_{5}$ is the supremum of $\tau \in \mathbb{T}_{0}$ for which $\bar{E}_{2}(\tau)$ and the term multiplied by $\operatorname{ce}_{\mathrm{f}}\left(\tau, \tau_{0}\right)$ in (37) are positive. Furthermore, assume (i) $E_{2}(\tau)>0$ and (ii) $\bar{E}_{2}(\tau)>0$ and the term multiplied by ce $e_{\mathrm{f}}\left(\tau, \tau_{0}\right)$ in (37) is positive for all $\tau \in \mathbb{T}_{0}$; subsequently, inequalities (36) and (37) hold respectively for all $\tau \in \mathbb{T}_{0}$.

Proof (i) Let $0<\alpha<1$, and we define a function $\chi(\tau)$ by

$$
c+\int_{\tau_{0}}^{\tau} \mathrm{f}(s) v(s) \Delta s+\int_{\tau_{0}}^{\tau} \mathrm{g}(s) v^{\alpha}(s)\left[v(s)+\int_{\tau_{0}}^{s} h(x) v^{\alpha+1}(x) \Delta x\right] \Delta s,
$$

which is the right-hand side of (34). Then $\chi\left(\tau_{0}\right)=c, v(\tau) \leq \chi(\tau)$ and

$$
\chi^{\Delta}(\tau) \leq \mathrm{f}(\tau) \chi(\tau)+\mathrm{g}(\tau) \chi^{\alpha}(\tau)\left(\chi(\tau)+\int_{\tau_{0}}^{\tau} h(x) \chi^{\alpha+1}(x) \Delta x\right) .
$$

Define a function $\psi(\tau)$ by

$$
\psi(\tau)=\chi(\tau)+\int_{\tau_{0}}^{\tau} h(x) \chi^{\alpha+1}(x) \Delta x .
$$

From (38) and (39) and the fact $\chi(\tau) \leq \psi(\tau)$ it follows that

$$
\psi^{\Delta}(\tau) \leq \mathrm{f}(\tau) \psi(\tau)+(\mathrm{g}(\tau)+h(\tau)) \psi^{\alpha+1}(\tau) .
$$

Now the application of Theorem 3.1 to inequality (40) yields

$$
\psi(\tau) \leq c e_{\mathrm{f}}\left(\tau, \tau_{0}\right)\left(E_{2}(\tau)\right)^{-\frac{1}{\alpha}} .
$$


Using (41) in (38), we get

$$
\chi^{\Delta}(\tau) \leq \mathrm{f}(\tau) \chi(\tau)+\left[c e_{\mathrm{f}}\left(\tau, \tau_{0}\right)\left(E_{2}(\tau)\right)^{-\frac{1}{\alpha}} \cdot \mathrm{g}(\tau)\right] \chi^{\alpha}(\tau) .
$$

Again by applying Theorem 3.1 to inequality (42) implies the estimate for $\chi(\tau)$ such that

$$
\begin{aligned}
\chi(\tau) \leq & c e_{\mathrm{f}}\left(\tau, \tau_{0}\right)\left[1+(1-\alpha) c^{\alpha} \int_{\tau_{0}}^{\tau} e_{\mathrm{f}}\left(s, \tau_{0}\right)\left(E_{2}(s)\right)^{-\frac{1}{\alpha}}\right. \\
& \left.\times \mathrm{g}(s)\left(e_{\ominus \mathrm{f}}\left(\sigma(s), \tau_{0}\right)\right)^{1-\alpha} \Delta s\right]^{\frac{1}{1-\alpha}} .
\end{aligned}
$$

Using (43) and the fact $v(\tau) \leq \chi(\tau)$, we get the required inequality:

$$
v(\tau) \leq c e_{\mathrm{f}}\left(\tau, \tau_{0}\right)\left[1+(1-\alpha) c^{\alpha} \int_{\tau_{0}}^{\tau} e_{\mathrm{f}}\left(s, \tau_{0}\right)\left(E_{2}(s)\right)^{-\frac{1}{\alpha}} \mathrm{g}(s)\left(e_{\ominus \mathrm{f}}\left(\sigma(s), \tau_{0}\right)\right)^{1-\alpha} \Delta s\right]^{\frac{1}{1-\alpha}} .
$$

(ii) For $1<\alpha<\infty$, a function $\chi(\tau)$ is defined as follows:

$$
\chi(\tau)=c+\int_{\tau_{0}}^{\tau} \mathrm{f}(s) v(s) \Delta s+\int_{\tau_{0}}^{\tau} \mathrm{g}(s) v^{\alpha}(s)\left[v(s)+\int_{\tau_{0}}^{s} h(x) v^{\alpha+1}(x) \Delta x\right] \Delta s .
$$

Thereafter, applying a similar method to that used in the proof of the first case, it is observed that every one of the inequalities from (38) to (42) holds for $\tau_{0}<\tau<j_{5}$. Additionally, for $1<\alpha<\infty$, inequality (43) gives an estimate for $\chi(\tau)$ for $\tau_{0} \leq \tau<j_{5}$ in order that

$$
\begin{aligned}
\chi(\tau) \leq & c e_{\mathrm{f}}\left(\tau, \tau_{0}\right)\left[1-(\alpha-1) c^{\alpha} \int_{\tau_{0}}^{\tau} e_{\mathrm{f}}\left(s, \tau_{0}\right)\left(E_{2}(s)\right)^{-\frac{1}{\alpha}}\right. \\
& \left.\times \mathrm{g}(s)\left(e_{\ominus \mathrm{f}}\left(\sigma(s), \tau_{0}\right)\right)^{1-\alpha} \Delta s\right]^{\left(-\frac{1}{\alpha-1}\right)} .
\end{aligned}
$$

Using (44) and the fact $v(\tau) \leq \chi(\tau)$, the requisite inequality is achieved in (37). The final deduction is evident if the proofs of the first and second cases, mentioned above, are followed. The proof is complete.

\section{Remark 4.6}

- Theorems 4.1, 4.2, 4.3, and 4.5 reduce to Theorems 2.4.1, 2.4.5, 2.4.4, and 2.4.2, respectively, if $\mathbb{T}=\mathbb{Z}$. These were established by Pachpatte in [17].

- Similarly, for $\mathbb{T}=\mathbb{R}$, Theorems 4.1, 4.2, 4.3, and 4.5 reduce to Theorems 2.7.1, 2.7.2, 2.7.3, and 2.7.4, respectively. These were established by Pachpatte in [16].

\section{Applications}

Certain specific nonlinear integral inequalities play their part in the exploration of qualitative properties for solutions of certain differential, integral, and integro-differential equations [6]. This portion is related to the behavior of solutions of the higher order integrodynamic equations of the form

$$
y^{\Delta^{n}}+\sum_{i=1}^{n} \omega_{i}(\tau) y^{\Delta^{n-i}}=y^{\alpha} \mathrm{g}\left(\tau, y, \int_{\tau_{0}}^{\tau} h(\tau, s, y) \Delta s\right)
$$


by means of comparison with solutions of the dynamic equation

$$
y^{\Delta^{n}}+\sum_{i=1}^{n} \omega_{i}(\tau) y^{\Delta^{n-i}}=0,
$$

where $\omega_{i}: \mathbb{T}_{+} \rightarrow \mathbb{R}, h: \mathbb{T}_{+} \times \mathbb{T}_{+} \times \mathbb{T} \rightarrow \mathbb{R}, \mathrm{g}: \mathbb{T}_{+} \times \mathbb{T} \times \mathbb{T} \rightarrow \mathbb{R}$ are rd-continuous functions, $\sum_{k=1}^{n}(-\mu)^{k-1} \omega_{k}$ is regressive, and $0<\alpha<1$ is a constant. A considerable amount of studies of various general and special versions of equation (45) have been done, and many results have emerged in the literature. The theorems proved in the following present some results which are established by the application of the special version of the inequality given in Theorem 4.3. Here it will be assumed that every solution $y(\tau)$ of (45) under discussion exists on $\mathbb{T}_{+}$. In what follows, $y_{1}(\tau), \ldots, y_{n}(\tau)$ is a fundamental system for (46). Wronskian of $n$ functions $y_{1}(\tau), \ldots, y_{n}(\tau)$, all of them $n-1$ times differentiable, is defined naturally by

$$
W\left(y_{1}, \ldots, y_{n}\right)(\tau)=\operatorname{det}\left(\begin{array}{cccc}
y_{1} & y_{2} & \cdots & y_{n} \\
y_{1}^{\Delta} & y_{2}^{\Delta} & \cdots & y_{n}^{\Delta} \\
\cdot & \cdot & \cdot & \cdot \\
\cdot & \cdot & \cdot & \cdot \\
\cdot & \cdot & \cdot & \cdot \\
y_{1}^{\Delta n-1} & y_{2}^{\Delta-1} & \cdots & y_{n}^{\Delta^{n-1}}
\end{array}\right) \text {, }
$$

and $W(\tau, s)$ is the determinant of the matrix in (47) where the last row is replaced by $\left(y_{1}(s) \cdots y_{n}(s)\right)$. The following result deals with the boundedness of the solution of (45) under some suitable conditions on the functions involved in (45) and on the solutions of equation (46).

\section{Theorem 5.1 Suppose that}

$$
\left|\sum_{k=1}^{n} c_{k} y_{k}(\tau)\right| \leq c, \quad \tau \in \mathbb{T}_{+},
$$

where $c_{1}, \ldots, c_{n}$ and $c>0$ are constants.

$$
\left|\frac{W(\sigma(s), \tau)}{W(\sigma(s))}\right| \leq M, \quad 0 \leq s \leq \tau<\infty
$$

$M>0$. Let the functions $\mathrm{g}$ and $\mathrm{h}$ in (45) be bounded by

$$
\begin{aligned}
& |g(\tau, y, z)| \leq a(\tau)(|y|+|z|), \quad \tau \in \mathbb{T}_{+} \\
& |h(\tau, s, y)| \leq b(s)|y|, \quad 0 \leq s \leq \tau<\infty
\end{aligned}
$$

where $a, b: \mathbb{T}_{+} \rightarrow \mathbb{R}_{+}$are rd-continuous functions. If

$$
E_{1}(\tau)=1-c^{\alpha} \alpha M \int_{\tau_{0}}^{\tau} a(s) e_{\alpha b}\left(s, \tau_{0}\right) \Delta s>0
$$


for all $\tau \in \mathbb{T}_{+}$and

$$
\int_{\tau_{0}}^{\infty} a(s)\left(E_{1}(s)\right)^{-\frac{1}{\alpha}} e_{b}\left(s, \tau_{0}\right) \Delta s<\infty
$$

then all solutions of $(45)$ are bounded on $\mathbb{T}_{+}$.

Proof By using the variations of constants formula from Theorem 5.119 in [4], the solutions of (45) and (46) are related by the integral equation

$$
y(\tau)=\sum_{i=1}^{n} c_{i} y_{i}+\int_{\tau_{0}}^{\tau} \frac{W(\sigma(s), \tau)}{W(\sigma(s))} y^{\alpha}(s) \mathrm{g}\left(s, y(s), \int_{\tau_{0}}^{s} h(s, t, y(t)) \Delta t\right) \Delta s,
$$

where $y_{1}, \ldots, y_{n}$ are linearly independent solutions of (46). From (54) and (48)-(51) we obtain

$$
|y(\tau)| \leq c+\int_{\tau_{0}}^{\tau} M a(s)|y(s)|^{\alpha}\left(|y(s)|+\int_{\tau_{0}}^{s} b(t)|y(t)| \Delta t\right) \Delta s .
$$

On application of a particular form of Theorem 4.3 (i.e., when $f(\tau)=0$ ), we will get the following:

$$
|y(\tau)| \leq c\left[1+(1-\alpha) c^{\alpha} M \int_{\tau_{0}}^{\tau} a(s)\left(E_{1}(s)\right)^{-\frac{1}{\alpha}} e_{b}\left(s, \tau_{0}\right) \Delta s\right]^{\frac{1}{1-\alpha}} .
$$

The approximation from the above inequality and hypothesis (53) indicate the boundedness of all the solutions of (45) on $\mathbb{T}_{+}$.

The upcoming theorem illustrates that taking into account certain appropriate circumstances on the functions involved in (45) and on the solutions of equation (46), all the solutions of (45) tend towards zero as $\tau$ tends towards $\infty$.

\section{Theorem 5.2 Suppose that}

$$
\left|\sum_{k=1}^{n} c_{k} y_{k}(\tau)\right| \leq c e_{-\beta}\left(\tau, \tau_{0}\right), \quad \tau \in \mathbb{T}_{+}
$$

where $c_{1}, \ldots, c_{n}$ and $c>0$ are constants.

$$
\left|\frac{W(\sigma(s), \tau)}{W(\sigma(s))}\right| \leq M e_{-\beta}(\tau, s), \quad 0 \leq s \leq \tau<\infty,
$$

$M>0$. Let the functions $\mathrm{g}$ and $h$ in (45) be bounded by

$$
\begin{aligned}
& |\mathrm{g}(\tau, y, z)| \leq a(\tau)(|y|+|z|), \quad \tau \in \mathbb{T}_{+}, \\
& |h(\tau, s, y)| \leq e_{-\beta}\left(\tau, \tau_{0}\right) b(s)|y|, \quad 0 \leq s \leq \tau<\infty,
\end{aligned}
$$


where $a, b: \mathbb{T}_{+} \rightarrow \mathbb{R}_{+}$are rd-continuous functions. If

$$
E_{2}(\tau)=1-c^{\alpha} \alpha M \int_{\tau_{0}}^{\tau} e_{-\alpha \beta}\left(t, \tau_{0}\right) a(t)\left(e_{b(\zeta) e_{-\beta}\left(\zeta, \tau_{0}\right)}\left(\sigma(t), \tau_{0}\right)\right)^{\alpha} \Delta t>0
$$

for all $\tau \in \mathbb{T}_{+}$and

$$
\int_{\tau_{0}}^{\infty} a(s) e_{-\alpha \beta}\left(s, \tau_{0}\right)\left(E_{2}(s)\right)^{-\frac{1}{\alpha}} e_{b(t) e_{-\beta}\left(t, \tau_{0}\right)}\left(s, \tau_{0}\right) \Delta s<\infty
$$

as a consequence, the solutions of (45) tend towards zero as $\tau$ tends towards $\infty$.

Proof Let $y_{1}(\tau), \ldots, y_{n}(\tau)$ be linearly independent solutions of (46), then the solutions of (45) and (46) are related by the integral equation (54). Using (57)-(60), we obtain

$$
\begin{aligned}
|y(\tau)| \leq c e_{-\beta}\left(\tau, \tau_{0}\right) & +\int_{\tau_{0}}^{\tau} M e_{-\beta}(\tau, s) p(s)|y(s)|^{\alpha}\left(|y(s)|+e_{-\beta}\left(s, \tau_{0}\right) \int_{\tau_{0}}^{s} q(t)|y(t)| \Delta t\right) \Delta s, \\
|y(\tau)| e_{\beta}\left(\tau, \tau_{0}\right) \leq & c+\int_{\tau_{0}}^{\tau} M p(s) e_{-\alpha \beta}\left(s, \tau_{0}\right)\left(|y(s)| e_{\beta}\left(s, \tau_{0}\right)\right)^{\alpha} \\
& \times\left(|y(s)| e_{\beta}\left(s, \tau_{0}\right)+\int_{\tau_{0}}^{s} q(t) e_{-\beta}\left(t, \tau_{0}\right)|y(t)| e_{\beta}\left(t, \tau_{0}\right) \Delta t\right) \Delta s .
\end{aligned}
$$

On application of a particular form of Theorem 4.3 (i.e., when $\mathrm{f}(\tau)=0$ ) and taking $u(\tau)=$ $|y(\tau)| e_{\beta}\left(\tau, \tau_{0}\right)$, we obtain an inequality which on multiplication by $e_{-\beta}\left(\tau, \tau_{0}\right)$ will provide the following:

$$
\begin{aligned}
|y(\tau)| \leq & c e_{-\beta}\left(\tau, \tau_{0}\right)\left[1+(1-\alpha) c^{\alpha} \int_{\tau_{0}}^{\tau} M p(s) e_{-\alpha \beta}\left(s, \tau_{0}\right)\left(E_{2}(s)\right)^{-\frac{1}{\alpha}}\right. \\
& \left.\times e_{q(t) e_{-\beta}\left(t, \tau_{0}\right)}\left(s, \tau_{0}\right) \Delta s\right]^{\frac{1}{1-\alpha}} .
\end{aligned}
$$

Taking in view hypothesis (62), the preceding approximation provides the required result.

Definition 5.3 For a slowly growing function $F(\tau)$, where $F(\tau)$ is continuous, there exists a constant $\Lambda$ for every $\epsilon>0$ such that

$$
|\mathrm{F}(\tau)| \leq \Lambda e_{\epsilon}\left(\tau, \tau_{0}\right), \quad \tau \in \mathbb{T}_{0}
$$

where $\Lambda$ may depend on $\epsilon$.

The succeeding outcome signifies that compared to any positive exponential function, all the solutions of (45) grow more slowly.

Theorem 5.4 Suppose that

$$
\left|\sum_{k=1}^{n} c_{k} y_{k}(\tau)\right| \leq c e_{\beta}\left(\tau, \tau_{0}\right), \quad \tau \in \mathbb{T}_{+},
$$


where $c_{1}, \ldots, c_{n}$ and $c>0$ are constants.

$$
\left|\frac{W(\sigma(s), \tau)}{W(\sigma(s))}\right| \leq M e_{\beta}(\tau, s), \quad 0 \leq s \leq \tau<\infty,
$$

$M>0$. Let the functions $\mathrm{g}$ and $\mathrm{h}$ in (45) be bounded by

$$
\begin{array}{ll}
|\mathrm{g}(\tau, y, z)| \leq a(\tau)(|y|+|z|), & \tau \in \mathbb{T}_{+}, \\
|h(\tau, s, y)| \leq e_{\beta}\left(\tau, \tau_{0}\right) b(s)|y|, & 0 \leq s \leq \tau<\infty,
\end{array}
$$

where $a, b: \mathbb{T}_{+} \rightarrow \mathbb{R}_{+}$are rd-continuous functions. If

$$
E_{3}(\tau)=1-c^{\alpha} \alpha M \int_{\tau_{0}}^{\tau} e_{\alpha \beta}\left(t, \tau_{0}\right) a(t)\left(e_{b(\zeta) e_{-\beta}\left(\zeta, \tau_{0}\right)}\left(\sigma(t), \tau_{0}\right)\right)^{\alpha} \Delta t>0
$$

for all $\tau \in \mathbb{T}_{+}$and

$$
\int_{\tau_{0}}^{\infty} a(s) e_{\alpha \beta}\left(s, \tau_{0}\right)\left(E_{3}(s)\right)^{-\frac{1}{\alpha}} e_{b(t) e_{-\beta}\left(t, \tau_{0}\right)}\left(s, \tau_{0}\right) \Delta s<\infty
$$

results in proving that the solutions of (45) are growing steadily.

The above theorem can be easily proved by following an equivalent reasoning, which was followed to prove Theorem 5.2, with suitable modifications.

\section{Conclusion}

It has been shown that by using mean value theorem, a fundamental dynamic inequality is justifiable on any arbitrary time scale. The cases for some special time scales are as follows: For $\mathbb{T}=\mathbb{Z}$, the inequality coincides with the fundamental finite difference inequality presented by Pachpatte in ([17], Theorem 2.3.4). For $\mathbb{T}=\mathbb{R}$, we make an addition with a new fundamental integral inequality in classical analysis and use it in turn to reproduce the proofs of nonlinear integral Pachpatte's inequalities ([16], Theorems 2.7.1-2.7.4), which were formerly accomplished by using basic analytical approach.

For nonlinear dynamic Pachpatte's inequalities, we concluded that: If we take the time scale $\mathbb{T}=\mathbb{Z}$, the inequalities coincide with the fundamental finite difference inequalities presented by Pachpatte in ([17], Theorems 2.4.1, 2.4.2, 2.4.4, 2.4.5), and for $\mathbb{T}=\mathbb{R}$, the inequalities matched with nonlinear integral Pachpatte's inequalities produced in ([16], Theorems 2.7.1-2.7.4) by Pachpatte in the continuous setting. The preset work distinguishes from the existing ones in three aspects. Firstly, nonlinear Pachpatte's inequalities which we discussed here are quite different from the existing ones. Secondly, the existing literature about integral Pachpatte's inequality utilized comparison theorem to establish their inequalities and other results on time scales. But it is not helpful in our assertion and needs a more generalized form of it to obtain our desire estimates. Finally, in the applied sense, the existing manuals cover first order dynamic equations, while the feasibility of our derived estimates is shown in first as well as in higher order dynamic equations. 
Competing interests

It is declared that no competing interests exist regarding this manuscript.

\section{Authors' contributions}

All authors have equal contribution in this manuscript. All authors have checked and approved the final version.

\section{Author details}

'Department of Mathematics, Institute of Business Administration (IBA), Karachi, Pakistan. ${ }^{2}$ Department of Mathematics and Statistics, University of Swat, Khyber Pakhtunkhwa, Pakistan.

\section{Publisher's Note}

Springer Nature remains neutral with regard to jurisdictional claims in published maps and institutional affiliations.

Received: 23 March 2019 Accepted: 9 September 2019 Published online: 23 September 2019

\section{References}

1. Agarwal, R., Bohner, M., Peterson, A.: Inequalities on time scales, a survey. Math. Inequal. Appl. 4, 535-557 (2001)

2. Bohner, E.A., Bohner, M., Akin, F.: Pachpatte inequalities on time scales. J. Inequal. Pure Appl. Math. 6, Article ID 6 (2005)

3. Bohner, M., Georgiev, S.G.: Multivariable Dynamic Calculus on Time Scales. Springer, Cham (2016)

4. Bohner, M., Peterson, A.: Dynamic Equations on Time Scales: An Introduction with Applications. Birkhäuser, Bostan (2001)

5. Bohner, M., Peterson, A.: Advances in Dynamic Equations in Time Scales. Birkhäuser, Bostan (2003)

6. Diblík, J., Rưžičková, M., Václavíkov, B.: Bounded solutions of dynamic equations on time scales. Int. J. Difference Equ. 3, 61-69 (2008)

7. Du, L., Xu, R.: Some new Pachpatte type inequalities on time scales and their applications. J. Math. Inequal. 6, 229-240 (2012)

8. Gu, J., Meng, F.W.: Some new nonlinear Volterra-Fredholm type dynamic integral inequalities on time scales. Appl. Math. Comput. 245(1), 235-242 (2014)

9. Hilger, S.: Analysis on measure chains-a unified approach to continuous and discrete calculus. Results Math. 18, 18-56 (1990)

10. Li, W.N.: Some Pachpatte type inequalities on times scales. Comput. Math. Appl. 57, 275-282 (2009)

11. Li, W.N., Sheng, W.: Some nonlinear integral inequalities on time scales. J. Inequal. Appl. 2007, 70465 (2007)

12. Liu, H., Meng, F.: Nonlinear retarded integral inequalities on time scales and their applications. J. Math. Inequal. 12 219-234 (2018)

13. Ma, Q.-H., Wang, J.-W., Ke, X.-H., Pečarić, J.: On the boundedness of a class of nonlinear dynamic equations of second order. Appl. Math. Lett. 26(11), 1099-1105 (2013)

14. Nasser, B.B., Boukerrioua, M.A., Defoort, M., Djemia, M., Hammami, M.A.: Some generalizations of Pachpatte inequalities on time scales. IFAC-PapersOnLine 50(1), 14884-14889 (2017)

15. Nasser, B.B., Boukerrioua, M.A., Defoort, M., Djemia, M., Hammami, M.A., Laleg-Kirati, T.: Sufficient conditions for uniform exponential stability and h-stability of some classes of dynamic equations on arbitrary time scales. Nonlinear Anal. Hybrid Syst. 32(1), 54-64 (2019)

16. Pachpatte, B.G.: Inequalities for Differential and Integral Equations. Academic Press, London (1998)

17. Pachpatte, B.G.: Inequalities for Finite Difference Equations. Dekker, New York (2002)

18. Pachpatte, D.B.: On a nonlinear dynamic integrodifferential equation on time scales. J. Appl. Anal. 16, 279-294 (2010)

19. Saker, S.H.: Some nonlinear dynamic inequalities on time scales and applications. J. Math. Inequal. 4(4), 561-579 (2010)

20. Saker, S.H.: Some nonlinear dynamic inequalities on time scales. Math. Inequal. Appl. 14(3), 633-645 (2011)

21. Sarikaya, M.Z., Ozkan, U.M., Yildirim, H.: Time scale integral inequalities similar to Qi's inequality. J. Inequal. Pure Appl. Math. 7(4), Article ID 128 (2006)

22. Sun, Y.G., Hassan, T.: Some nonlinear dynamic integral inequalities on time scales. Appl. Math. Comput. 220(4), 221-225 (2013)

\section{Submit your manuscript to a SpringerOpen ${ }^{\circ}$ journal and benefit from:}

- Convenient online submission

$\checkmark$ Rigorous peer review

- Open access: articles freely available online

- High visibility within the field

- Retaining the copyright to your article

Submit your next manuscript at springeropen.com 